\title{
Synthesis of dispersible nanosheets based on monolayer clays with imidazolium and ammonium cations having long-chain alkyl groups
}

\author{
Ayami SUZUKI, Yuya OAKI and Hiroaki IMAI ${ }^{\dagger}$ \\ Department of Applied Chemistry, Faculty of Science and Technology, Keio University, \\ 3-14-1 Hiyoshi, Kohoku-ku, Yokohama 223-8522, Japan
}

\begin{abstract}
Clay-based nanosheets were produced from montmorillonite modified with organic cations. Exfoliation of the clay was achieved by ion exchange with imidazolium and ammonium cations having long-chain alkyl groups. Dispersible nanosheets as a monolayer clay was obtained through the exfoliation by adjusting of the alkyl-chain length. Homogeneous dispersion of the nanosheets was demonstrated in a non-protonic polar liquid and polymeric solid media.

(C2017 The Ceramic Society of Japan. All rights reserved.
\end{abstract}

Key-words : Nanosheet, Clay, Montmorillonite, Nanocomposite

[Received December 2, 2016; Accepted February 12, 2017]

\section{Introduction}

Polymer-clay composite materials exhibit improved gas barrier properties, ${ }^{1)-3)}$ enhanced mechanical properties, ${ }^{1), 4), 5)}$ higher heat resistivity, 1),6) and excellent flame retardancy. 1),7) Dispersible thinly layered clays that are obtained through ion exchange by organic cations (organo-clays) are important as an excellent filler in designing the polymer-clay composites, because clays are abundant in the earth and have high thermal stability. However, the state of dispersed organo-clays, including their thickness in liquid and solid media, has not been studied sufficiently. Revealing the structure and properties of organo-clays is essential for the understanding and improvement of plastic-clay composite materials. In the present study, we characterized the organo-clays in organic liquid and solid media by changing the alkyl chain length as a modifying agent.

Montmorillonite (MMT), a typical clay mineral, is composed of aluminosilicate sheets consisting of octahedral and tetrahedral units of silicon, aluminum, magnesium, and oxygen atoms. Sodium ions existing between the negatively charged aluminosilicate layers are easily replaced with other inorganic and organic cations. ${ }^{8-11)}$ Fabrication of organo-clays from MMT is achieved through organification and thinning by ion exchange and swelling of the interlayers. ${ }^{12)-15)}$ Various properties of organic polymers, such as polyamide, are improved by the addition of a small amount of organo-clays. ${ }^{16)}$ In recent years, it has been shown that dispersion of clays as a monolayer in a polymer matrix is required to maintain their transparency for optics applications. The polymerization of monomers inserted into the interlayers of MMT provides a nanocomposite in which the clays are dispersed as a monolayer. ${ }^{17)}$ However, the polymerization technique cannot be applied to a wide variety of composite materials. The heat resistance of polymer-clay composites depends on the thermal properties of the polymeric molecule and organic cations modifying the clays. For complexation with high-heat-resistant resins, such as polyimide, polyarylate, polyether sulfone, and polycar-

Corresponding author: H. Imai; E-mail: hiroaki@applc.keio.ac.jp bonate, organic cations having a high heat resistance are needed for the modification of clays. Thus, we studied the fabrication method of monolayer clays that are dispersible in organic liquid media by various kinds of organic cations for the production of highly functional nanocomposite materials.

In the current work, we replaced sodium ions in the interlayer of MMT with ammonium ions and imidazolium ions having long-chain alkyl groups (Fig. 1). Dispersible nanosheets were then produced as a monolayer clay exfoliated in a non-protonic polar liquid. Homogeneous dispersion of the nanosheets was demonstrated in the liquid and polyimide media for application to polymer-clay nanocomposites.

\section{Experimental}

A certain amount $(6.0 \mathrm{~g})$ of montmorillonite (MMT) $\left[\mathrm{Na}_{2 / 3^{-}}\right.$ $\mathrm{Si}_{8}\left(\mathrm{Al}_{10 / 3} \mathrm{Mg}_{2 / 3} \mathrm{O}_{20} \cdot \mathrm{OH}\right)_{4}$ ] [Kunipia-F, cation exchange capacity (CEC): $115 \mathrm{mmol} / 100 \mathrm{~g}$, Kunimine] was added with stirring and dispersed into $300 \mathrm{~cm}^{3}$ of purified water using a $500 \mathrm{~cm}^{3}$ threenecked flask. The clay dispersion was stirred overnight at $80^{\circ} \mathrm{C}$. Ammonium salt or imidazolium salt having various alkyl chains (1.2 equivalent with respect to CEC) was dissolved into a mixture of $10 \mathrm{~cm}^{3}$ of purified water and $5 \mathrm{~cm}^{3}$ of ethanol. The solution was added dropwise to the clay dispersion and stirred for $2 \mathrm{~h}$ at $80^{\circ} \mathrm{C}$. We used $n$-decyl trimethyl ammonium chloride [DTMAMCL, number of carbons in the alkyl chain $\left(n_{\mathrm{C}}\right)=10$ ] and trimethylstearyl ammonium chloride (TMSAMCL, $n_{\mathrm{C}}=18$ ) as an ammonium salt and 1-methyl-3-decyl imidazolium chloride (DMIMCL, $n_{\mathrm{C}}=10$ ), 1-methyl-3-octyl imidazolium chloride (MOIMCL, $n_{\mathrm{C}}=8$ ), 1-hexyl-3-methylimidazolium chloride (HMIMCL, $n_{\mathrm{C}}=6$ ), and 1-butyl-3-methylimidazolium chloride $\left(\mathrm{BMIMCL}, n_{\mathrm{C}}=4\right)$ as an imidazolium salt. The chemical formulas of the organic cations are shown in Fig. 2.

The suspension was centrifuged after being cooled to room temperature. The resultant suspension was centrifuged again by adding purified water. This process was repeated three times to remove salts formed by the ion-exchange reaction from the dispersion. The third supernatant containing the ion-exchanged clay was washed twice with acetone. The clays were redispersed into 
(a)

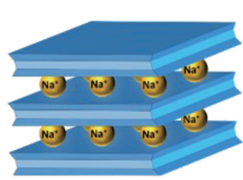

(c)

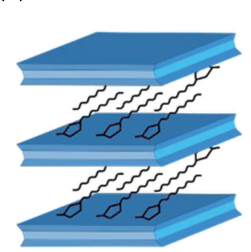

$(\mathrm{d})$

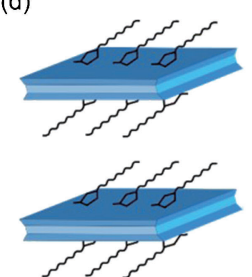

Fig. 1. A schematic illustration of the fabrication of monolayer clay from MMT by the exchange of sodium ions by organic cations in the interlayer; (a) MMT, (b) organo-clay after the exchange of sodium ions by organic cations having short alkyl chains, (c) organo-clay after the exchange by organic cations having long alkyl chains, and (d) monolayer clays covered with organic cations having long alkyl chains in liquid and polymer media.

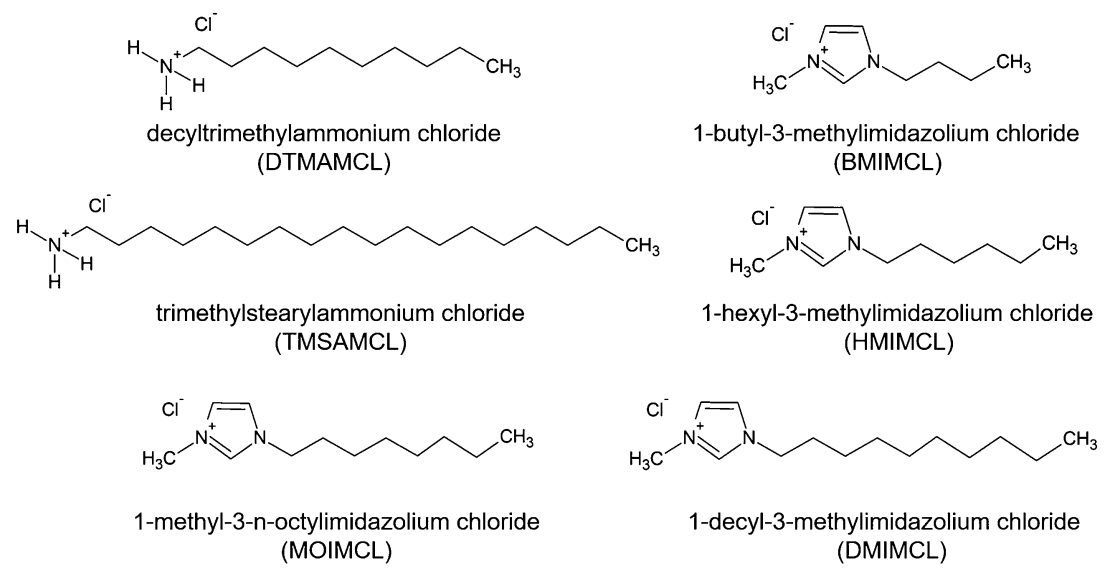

Fig. 2. Structural formulas of ammonium salts and imidazolium salts used in the present study.

N-methylpyrrolidone (NMP), which is generally used as a solvent of polyimide, and sonicated in an ultrasonic bath for more than $2 \mathrm{~h}$. Homogeneous dispersion of the clays in NMP was obtained after the removal of coarse particles by centrifugation. Polyimide-clay composite films were produced by casting a mixture of a polyimide varnish and the NMP dispersion on a glass plate. The ratio of clay and polyimide was $5 \mathrm{wt} \%$. A polyimide varnish (polyamic acid) was prepared from $4,4^{\prime}$-oxydianiline (ODA) and 1,2,4,5-benzenetetracarboxylic anhydride (PMDA). ODA $(3.0 \mathrm{~g})$ was dissolved in NMP $(25.0 \mathrm{~g})$ in a three-neck flask with a nitrogen line at $50^{\circ} \mathrm{C}$. A mixture of PMDA (3.3 g) and NMP $(27.2 \mathrm{~g})$ was gradually added to the amine solution at $70^{\circ} \mathrm{C}$. The polyimide varnish ( $5 \mathrm{wt} \%)$ obtained by the addition of NMP was cast with the NMP dispersion of the clay. Imidization of the mixture was performed at $80^{\circ} \mathrm{C}$ for $10 \mathrm{~min}$ and at $250^{\circ} \mathrm{C}$ for $30 \mathrm{~min}$.

Dried clays after the ion-exchange reaction were analyzed with X-ray diffraction (XRD) using a Rigaku Ultima IV diffractometer and Fourier transform infrared absorption (FTIR) using a Thermo Scientific Nicolet 6700FTIR. The ion-exchange rate of the clays was calculated from the mass change estimated by thermogravimetry (TG) using a Rigaku Thermo plus TGS8120. Exfoliated clays in the NMP dispersion were characterized by atomic force microscopy (AFM) using a Shimadzu SPM9600 after casting on a silicon substrate. Microtome sections of the polyimide-clay composite film were observed by transmission electron microscopy (TEM) using an FEI Tecnai F20.

\section{Results and discussion}

Figures 3 and 4 show the FTIR spectra and XRD patterns of MMTs before and after the ion-exchange reactions, respectively. From the presence of specific absorption bands in FTIR spectra

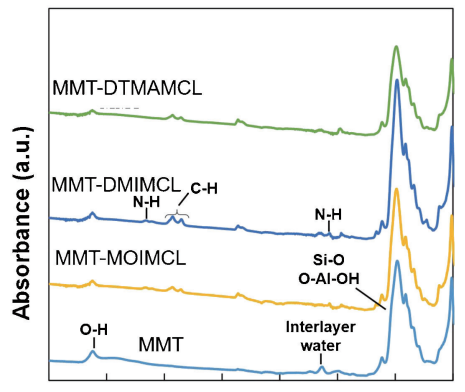

4000350030002500200015001000500

Wavenumber $\left(\mathrm{cm}^{-1}\right)$

Fig. 3. FTIR spectra of MMTs before and after ion exchange.

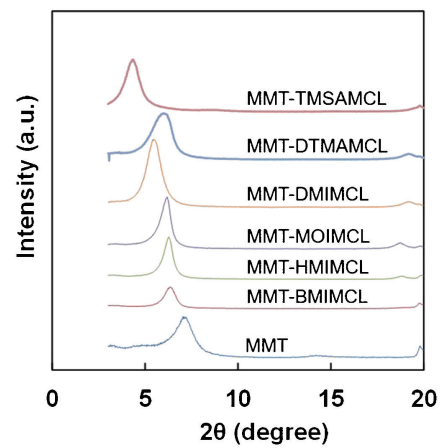

Fig. 4. XRD patterns of MMTs before and after ion exchange.

and the shift of a diffraction band assigned to the interlayer distance in XRD patterns, we confirmed the formation of organoclays that were MMTs containing organic cations in the inter- 
layers by the ion-exchange reaction. Hereafter, the organo-clays formed from MMTs with DTMAMCL $\left(n_{\mathrm{C}}=10\right)$, TMSAMCL $\left(n_{\mathrm{C}}=18\right)$, DMIMCL $\left(n_{\mathrm{C}}=10\right)$, MOIMCL $\left(n_{\mathrm{C}}=8\right)$, HMIMCL $\left(n_{\mathrm{C}}=6\right)$, and BMIMCL $\left(n_{\mathrm{C}}=4\right)$ are referred to as MMTDTMAMCL, MMT-TMSAMCL, MMT-DMIMCL, MMTMOIMCL, MMT-HMIMCL, and MMT-BMIMCL, respectively.

As shown in Fig. 3, the absorption bands of MMTs around 3600,1650 , and $1000 \mathrm{~cm}^{-1}$ are assigned to the $\mathrm{O}-\mathrm{H}$ bond, interlayer water, and the $\mathrm{Si}-\mathrm{O}$ and $\mathrm{O}-\mathrm{Al}-\mathrm{OH}$ bonds, respectively. ${ }^{18)}$ These main bands were not changed by the ion exchange, although the signal of interlayer water was relatively weak for the ion-exchanged clays. From the appearance of absorption bands around $2900 \mathrm{~cm}^{-1}$, which are ascribed to the $\mathrm{C}-\mathrm{H}$ bond, we found that organic molecules were contained in all of the ionexchanged clays. The absorption bands around 3150 and 1550 $\mathrm{cm}^{-1}$, which belong to the $\mathrm{N}-\mathrm{H}$ bond, indicate the presence of imidazolium cations in the interlayer of MMT.

The ion exchange rate of the organo-clays was evaluated from the mass decrease with the combustion of organic compounds using TG measurement (Fig. 5). We obtained the ion exchange rate (Table 1) from the mass ratio of the organic cations by comparison with the theoretical value, which was calculated by replacing $100 \mathrm{~mol} \%$ sodium ions in the interlayers. In the present system, more than $80 \mathrm{~mol} \%$ of sodium cations were found to be replaced by the organic cations in all of the organo-clays. The onset temperature of combustion for imidazolium cations, 270$349^{\circ} \mathrm{C}$, was higher than that for the ammonium cations, $227-$ $243^{\circ} \mathrm{C}$, in the organo-clays. This means that the imidazolium salts have a higher heat resistivity as a modifying agent for polymerclay composites.

As shown in the XRD patterns (Fig. 4), a diffraction peak of

Table 1. The diffraction angle, interlayer distance, ion exchange rate, and dispersibility of MMTs before and after ion exchange

\begin{tabular}{lcccc}
\hline \multicolumn{1}{c}{ Sample } & $20 \theta\left[^{\circ}\right]$ & $\mathrm{d}[\mathrm{nm}]$ & $\begin{array}{c}\text { Ion exchange rate } \\
\text { for CEC }\end{array}$ & $\begin{array}{c}\text { Dispersibility } \\
\text { in NMP }\end{array}$ \\
\hline MMT & 7.14 & 1.24 & - & - \\
MMT-BMIMCL & 6.36 & 1.39 & $90.5 \%$ & - \\
MMT-HMIMCL & 6.28 & 1.41 & $80.2 \%$ & - \\
MMT-MOIMCL & 6.18 & 1.43 & $81.0 \%$ & $\mathrm{D}$ \\
MMT-DMIMCL & 5.48 & 1.61 & $88.7 \%$ & $\mathrm{D}$ \\
MMT-DTMAMCL & 6.12 & 1.44 & $84.6 \%$ & $\mathrm{D}$ \\
MMT-TMSAMCL & 4.34 & 2.04 & $95.2 \%$ & - \\
\hline
\end{tabular}

around $7^{\circ}$ assigned to the interlayer distance of MMTs was shifted into a lower angle by the exchange of organic cations. ${ }^{19), 20)}$ Since more than $80 \mathrm{~mol} \%$ of sodium cations were replaced by the organic cations in all of the organo-clays, the interlayer distance is deduced to be mainly affected by the alkyl chain length. Table 1 lists the diffraction angle and corresponding interlayer distance for the clays before and after the ion exchange by various organic cations. The interlayer distance of MMTs was increased from 1.39 to $1.61 \mathrm{~nm}$ by imidazolium cations upon increasing the alkyl chain length $\left(n_{\mathrm{C}}\right)$ from 4 to 10 . However, the variation in distance was small in the cations having $n_{\mathrm{C}}=4,6$, and 8 . This suggests that the cationic molecules having short alkyl chains are arranged parallel to the aluminosilicate layers [Fig. 1(b)]. On the other hand, the interlayer distance was increased to 1.44 and $2.04 \mathrm{~nm}$ by ammonium cations with $n_{\mathrm{C}}=10$ and 18 , respectively.

We achieved homogeneous dispersion by the sonication of organo-clays exchanged by DTMAMCL $\left(n_{\mathrm{C}}=10\right)$, DMIMCL $\left(n_{\mathrm{C}}=10\right)$, and MOIMCL $\left(n_{\mathrm{C}}=8\right)$ in NMP (Table 1). Other organo-clays were not dispersive in NMP and precipitation occurred immediately after centrifugation. Figure 6 shows AFM images of dispersive nanosheets obtained from the organo-clays on a silicon substrate by drying the homogeneous dispersion. The thicknesses of dispersive nanosheets obtained from MMTMOIMCL, MMT-DMIMCL, and MMT-DTMAMCL were 1.53, 1.31 , and $1.66 \mathrm{~nm}$, respectively. Because the thickness is in a range from 1 to $2 \mathrm{~nm}$, the nanosheets are deduced to be an aluminosilicate monolayer of MMT covered with the organic cations. These results suggest that the chain length of MOIMCL, DMIMCL, and DTMAMCL $\left(n_{\mathrm{C}}=8,10\right.$ and 10$)$ is suitable for

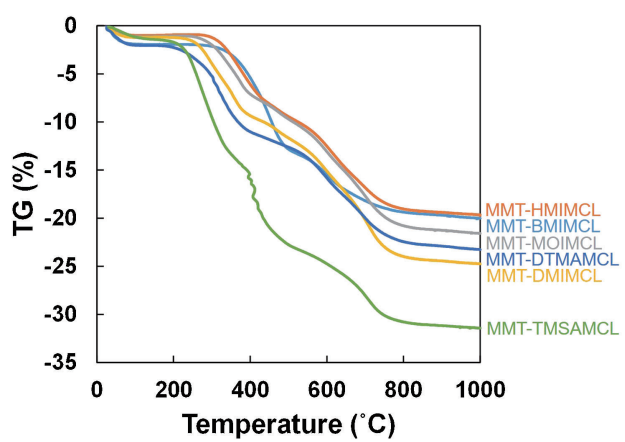

Fig. 5. TG curves of MMTs after ion exchange.
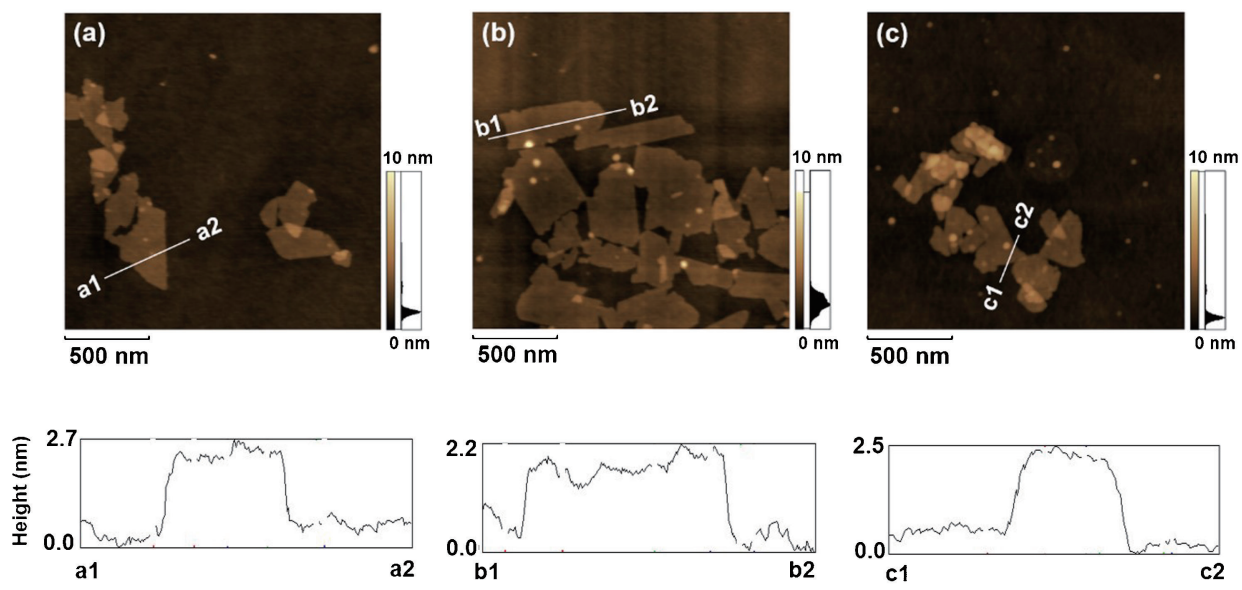

Fig. 6. AFM images and their height profiles of dispersive nanosheets obtained from MMT-MOIMCL (a), MMT-DMIMCL (b), and MMT-DTMAMCL (c). 

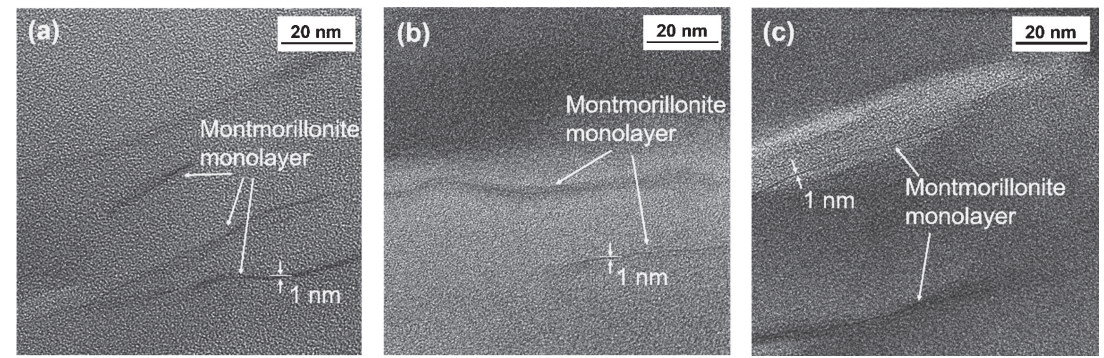

Fig. 7. TEM images of cross-section films of polyimide-clay composites obtained with MMT-MOIMCL (a), MMTDMIMCL (b), and MMT-DTMAMCL (c).

exfoliation of the organo-clays in the non-protonic polar liquid. The hydrophobicity of alkyl chains shorter than 8 is weak, and the affinity of an alkyl chain longer than 10 to the polar medium is low. Thus, the optimal chain length of the modifying agent is required for the production of dispersive monolayer clays.

Figure 7 shows TEM images of microtome sections of the polyimide-clay composite film. We clearly observed sheets $\sim 1 \mathrm{~nm}$ thick in the polymer matrix. Thus, the monolayer clays covered with organic cations were also dispersive in polyimide. Polyimide-clay composite films were produced by casting a mixture of a polyimide varnish and the NMP dispersion containing MMT-MOIMCL, MMT-DMIMCL, and MMT-DTMAMCL on a glass plate. The monolayer clays were easily arranged parallel to the glass surface by the casting technique. Thus, we observed the cross section of the monolayers in the polymer matrix of the microtome sections.

\section{Conclusion}

In the present study, we produced dispersible nanosheets as a monolayer clay exfoliated from montmorillonite by ion exchange with ammonium ions and imidazolium ions having long-chain alkyl groups. Homogeneous dispersion of nanosheets covered with organic cations was achieved in a non-protonic polar liquid and polymeric solid media. The preparation of clay-derived dispersive nanosheets is regarded as an important technique to improve various properties of polymer-clay nanocomposites.

\section{References}

1) M. Alexandre and P. Dubois, Mater. Sci. Eng., 28, 1-63 (2000).

2) K. Yano, A. Usuki and A. Okada, J. Polym. Sci. A Polym. Chem., 35, 2289-2294 (1997).
3) K. Yano, A. Usuki, A. Okada and N. Kurauchi, Jpn. Patent Koukai, 1992-33955 (1992).

4) Y. Kojima, A. Usuki, M. Kawasumi, A. Okada, Y. Fukushima, T. Kurauchi and O. Kamigaito, J. Mater. Res., 8, 1185-1189 (1993).

5) E. P. Giannelis, Adv. Mater., 8, 29-35 (1996).

6) A. Blumstein, J. Polym. Sci. A Polym. Chem., 3, 2665-2672 (1965).

7) J. W. Gilman, T. Kashiwagi and J. D. Lichtenhan, SAMPE J., 33, 40-46 (1997).

8) H. Takeshi and Y. Uno, J. Clay Sci. Soc. Jpn., 20, 67-78 (1980).

9) C. S. Ross and E. V. Shannon, J. Am. Ceram. Soc., 9, 77-96 (1926).

10) C. S. Ross and S. B. Hendricks, U.S. Geol. Surv. Prof. Pap., 205B, 23-79 (1945).

11) M. V. Eirish and L. I. Tret'yakova, Clay Miner., 8, 255-266 (1970).

12) H. Komine and N. Ogata, J. Jpn. Soc. Civ. Eng., 701, 373-385 (2002).

13) K. Norrish, Discuss. Faraday Soc., 18, 120-134 (1954).

14) S. Karaborni, B. Smit, W. Heidug, J. Urai and E. van Oort, Science, 271, 1102-1104 (1996).

15) R. W. Mooney, A. G. Keenan and L. A. Wood, J. Am. Chem. Soc., 74, 1371-1374 (1952).

16) N. Hasegawa, H. Okamoto, M. Kato, A. Usuki and N. Sato, Polymer, 44, 2933-2937 (2003).

17) Y. Kojima, A. Usuki, M. Kawasumi, A. Okada, T. Kurauchi and O. Kamigaito, J. Polym. Sci. A Polym. Chem., 31, 983-986 (1993).

18) S. Okuda and K. Inoue, J. Clay Sci. Soc. Jpn., 2, 61-75 (1962).

19) P. Maiti, K. Yamada, M. Okamoto, K. Ueda and K. Okamoto, Chem. Mater., 14, 4654-4661 (2002).

20) A. B. Morgan and J. W. Gilman, J. Appl. Polym. Sci., 87, 1329-1338 (2003). 